\title{
Facilitating effects of exercise on information processing
}

\author{
KAREN DAVRANCHE* and MICHEL AUDIFFREN \\ Laboratoire d'Analyse de la Performance Motrice Humaine (LAPMH), Maison des Sciences de l'Homme et de la \\ Société, 99 Avenue du Recteur Pineau, 86000 Poitiers, France
}

Accepted 8 November 2003

\begin{abstract}
The aim of this study was to examine the facilitating effects of moderate physical exercise on the reaction process to gain a better understanding of the interaction between physiological and cognitive processes. Sixteen participants with specific expertise in decision-making sports performed a double task consisting of choice reaction time while cycling. Signal quality, stimulus-response compatibility and time uncertainty were manipulated. Participants were tested at rest and while cycling at $20 \%$ and at $50 \%$ of their maximal aerobic power. A mood assessment questionnaire and a critical flicker fusion test were administered before and after the choice reaction time task. The results showed that moderate-intensity exercise $(50 \%$ maximal aerobic power) improves cognitive performance and that low-intensity exercise (20\% maximal aerobic power) enables participants to compensate the negative dual-task effect.
\end{abstract}

Keywords: additive factor method, arousal, cognitive-energetical model, critical flicker fusion, reaction time.

\section{Introduction}

In many sports (e.g. team sports, fighting sports and racket sports), players must simultaneously handle physiological and cognitive loads. A better understanding of the interaction between physiological and cognitive processes during exercise would be beneficial for practitioners. Indeed, it might be useful to improve training procedures and competitive sport strategies.

The effect of physical exercise on response performance has been studied using a wide variety of information-processing tasks (for comprehensive reviews, see Tomporowski and Ellis, 1986; McMorris and Graydon, 2000). According to the literature, the use of a systematic and rigorous methodology is necessary to enhance cognitive efficiency during exercise (Brisswalter and Legros, 1996; Brisswalter et al., 2002). Several factors should be considered in order to observe a facilitating effect of exercise on information processing: (1) the intensity and duration of the physical exercise, (2) the nature of the cognitive task, (3) the time when the psychological test is administrated and (4) the participant's ability at decision making. Studies of submaximal exercise have shown an improvement in cognitive performance during physical exercise (e.g. Brisswalter et al., 1994; McMorris and Graydon, 1996,

\footnotetext{
* Author to whom all correspondence should be addressed.
}

e-mail: karen.davranche@mshs.univ-poitiers.fr
1997; Arcelin et al., 1997, 1998). However, the duration of the exercise must be taken into account. Indeed, central and peripheral fatigue result in a performance decrement (Chmura et al., 1998; Guézennec, 2000). The duration of the exercise must be long enough to allow sufficient data to be collected and to stabilize the physiological parameters (longer than 3 min; Hagberg et al., 1978), yet not too long to avoid fatigue (shorter than $30 \mathrm{~min}$; Fischer et al., 1991). The physiological effects of the exercise intervention among participants would appear crucial to an unambiguous assessment of the effects of physical exercise on cognitive performance (Tomporowski and Ellis, 1986).

The use of individual relative workload is recommended in exercise psychology research. Using this framework, three predictors of exercise intensity have generally been used: maximal oxygen uptake, maximal aerobic power and the ventilatory threshold. In this study, workload was determined by a preliminary maximal aerobic test. Individual workloads were determined as a function of a percentage of maximal aerobic power for each participant.

Complex decision-making tasks are most appropriate for enhancing cognitive efficiency (Isaacs and Pohlman, 1991; McMorris and Keen, 1994; Brisswalter et al., 1997). It has been shown that simple tasks, such as simple reaction time tasks, do not always result in a facilitating effect of physical exercise. The timing of the administration of cognitive tests (during or after 
exercise) is also a decisive factor because physiological changes, such as central and plasma catecholamine concentrations, quickly return to basal values. Despite the fact that the dual-task paradigm may underestimate the facilitating effect of exercise, the use of simultaneous tasks is preferable.

Finally, the effect of exercise on cognitive performance varies according to the participant's expertise in decision-making sports (Delignières et al., 1994; McMorris and Graydon, 1996). It has been reported that sports that require decisions to be made under high energy expenditure may enhance the performance of experts in choice reaction time tasks during physical exercise more than that of non-experts. As a function of their expertise, the amount of resources invested in the task and the cognitive activity of the participants were presumably different (Delignières et al., 1994).

The purpose of this study was to advance our understanding of the interaction between physiological mechanisms and cognitive processes during exercise. More precisely, we wished to establish the exact nature of the facilitating effect of exercise on the reaction process within the framework of Sanders' $(1983,1998)$ cognitive-energetical model and Sternberg's (1969, 1998) additive factors method. Within this same framework, Arcelin et al. (1998) suggested an improvement in the third quartile of the reaction time distribution induced by the effect of exercise. However, these results were not obtained on absolute mean reaction time. In fact, Arcelin et al. (1998) observed an additive relation between physical exercise and stimulus-response compatibility, foreperiod duration and signal quality on mean reaction time. However, an additive effect means the absence of an interaction.
Analysis of variance, used within the framework of the additive factors method, does not allow for the evaluation of the risk of saying wrongly that there is no interaction. An additive effect between two factors can only be considered to be a real absence of an interaction if it proves to be robust (i.e. it is observed 95 times out of 100). In the present study, we wished to verify the additive effects and the interaction pattern observerd by Arcelin et al. (1998) but under different experimental conditions (intensity of exercise, exercise duration, number of trials, means of manipulating signal quality and foreperiod duration, number of alternatives).

Sanders' (1983) model is organized on three levels (Fig. 1). The first is composed of four informationprocessing stages functioning in a discrete serial mode. The stages are stimulus pre-processing, feature extraction, response choice and motor adjustment. The second level corresponds to three energetic attention control mechanisms principally brought to light by research in neurophysiology and neuropsychology: arousal, effort and activation (Pribram and McGuinness, 1975; McGuinness and Pribram, 1980). The mechanisms of arousal and activation are considered to be basal mechanisms. The effort mechanism represents a superior mechanism that supervises and coordinates the resource level of the basal mechanisms. Each of these mechanisms supplies a specific informationprocessing stage. The arousal mechanism receives energy by means of the stimulus pre-processing stage and supplies the feature extraction stage. The activation mechanism supplies the resources in the motor adjustment stage. Finally, the effort mechanism allocates resources to the response choice stage when the

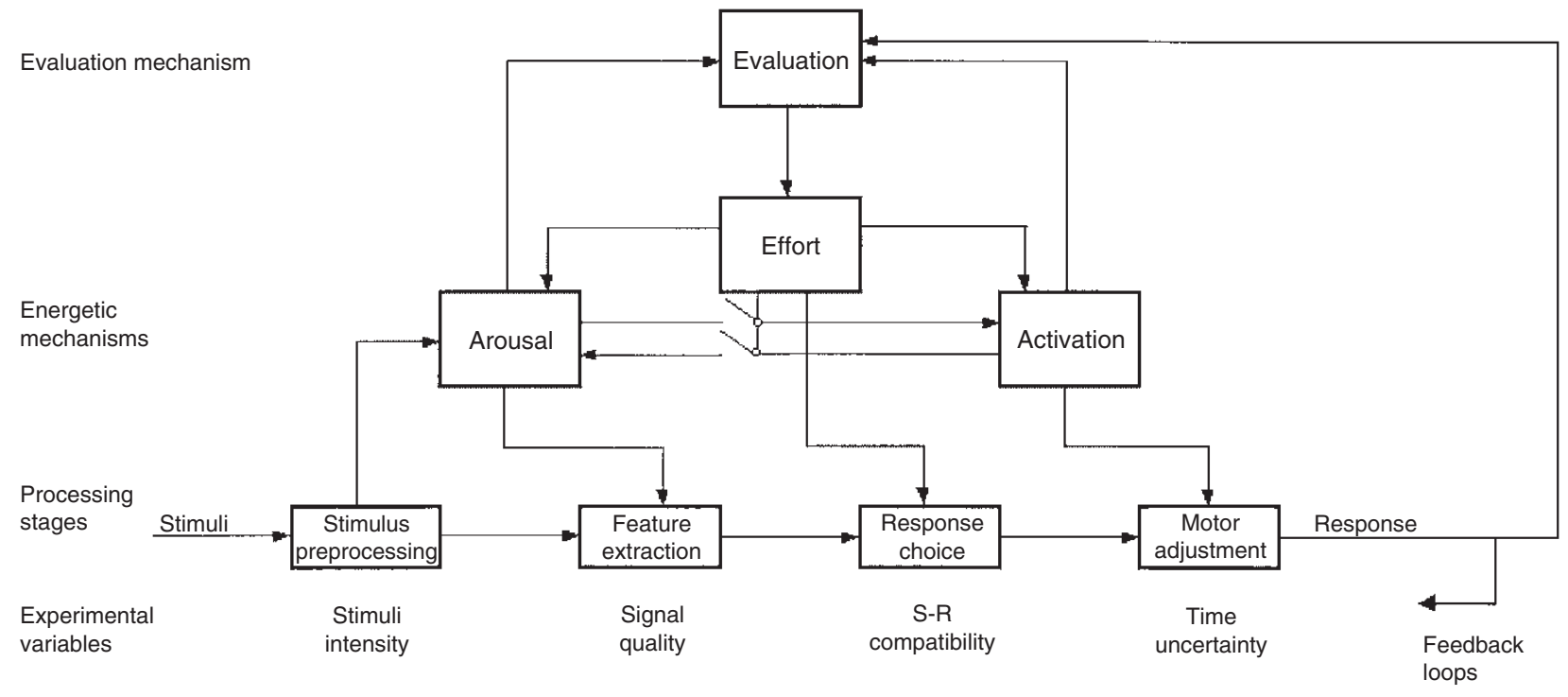

Fig. 1. Cognitive-energetical model (Sanders, 1983). S-R compatibility $=$ stimulus-response compatibility. 
decision-making task is complex to compensate a deficit of the two basal mechanisms. The effort mechanism is informed about the state of the basal mechanisms by an evaluation mechanism representing the third level of this model. To construct the cognitive-energetic model of stress and performance, Sanders $(1980,1983)$ based his work on numerous experimental results obtained using the additive factors method of Sternberg (1969).

Following the additive factors method, which is based on the discrete serial information-processing model, this method considers reaction time as the sum of the duration of each of the processing stages that occur between the moment of response signal and the initiation of a response. For each stage, at least one computational factor exists that directly and selectively affects its duration without modifying processing quality (assumption of selective influence). Computational factors that affect the stages of pre-processing, feature extraction, response choice and motor adjustment are, respectively, stimulus intensity, signal quality, stimulus-response compatibility and time uncertainty. In this study, only the three last factors were manipulated. Signal quality was manipulated by presenting intact or degraded response signals. Classically, reaction time is longer when the signal is degraded compared to when it is intact. This change in reaction time reflects a lengthening of perceptive processes and, more precisely, the feature extraction stage. Stimulusresponse compatibility was manipulated by changing the natural relationship between stimulus set and response set. Reaction time is longer when the relationship between the stimulus set and the response set is incompatible compared to when it is compatible. This change in reaction time reflects a lengthening of decisional processes and, more precisely, the response choice stage. Time uncertainty was manipulated by varying foreperiod duration; that is, the time between the warning auditory signal and the response signal. Reaction time is longer when foreperiod duration is long than when it is short. This change in reaction time reflects a lengthening of motor processes and, more precisely, the motor adjustment stage. The use of the additive factors method allows the determination of the number and nature of the stage(s) affected by one given factor (in our case, the physiological demand at rest or during exercise). According to Sternberg (1969), if a simple addition of the actions of two factors is observed, then it can be hypothesized that these two factors affect different stages; however, if an interaction between these two is observed, the variables are likely to affect at least one common processing stage.

As noted by Yagi et al. (1999), the use of reaction time as the sole measure of cognition limits the interpretation of the results. To gain a better understanding of the facilitating effect of physical exercise, we compared the results obtained with different variables. In addition to reaction time, we determined several other dependent variables: critical flicker fusion (Powell, 1982), subjective mood change (Thayer, 1989), heart rate and perceived exertion (DP-15; Delignières et al., 1994). As a measure of overall central nervous system activity, critical flicker fusion appears to be a simple and reliable way of assessing changes in cortical arousal (Smith and Misiak, 1976; Parkin et al., 1998). These changes, which are related to the retino-cortical system, can be considered to be linked to the arousal mechanism. Therefore, the critical flicker fusion test represented an additional dependent variable to infer an effect of physical exercise on the input processing stage. Subjective mood change (linked to alertness state) and heart rate can be considered to be indicators of a general activation of the organism. However, a distinction between action on the arousal mechanism and action on the activation mechanism is unclear. Finally, perceived exertion can be considered to be related to the effort required in the task (Arcelin et al., 1997).

The aim of this study was to assess the facilitating effect of submaximal exercise on cognitive processes. First, we expected to observe: (1) a degradation of cognitive performance during the $20 \%$ maximal aerobic power condition due to a negative dual-task effect; (2) an improvement of cognitive performance during the $50 \%$ maximal aerobic power condition induced by a facilitating effect of exercise; and (3) an increase in arousal and/or activation seen as a change in heart rate, subjective alertness and critical flicker fusion determination sensitivity.

Secondly, we expected to establish the exact nature of the effect of the exercise on reaction processes by localizing its facilitation effect through the observation of over-additive interaction(s) between physiological demands and experimental factors. Thus if exercise affects the feature extraction stage, we can predict that the signal quality effect is more important at rest than during exercise. Similarly, if exercise influences the response choice stage, we can expect the stimulusresponse compatibility effect to be larger at rest than during exercise. Finally, if exercise affects the motor adjustment stage, we can predict that the foreperiod duration effect is more pronounced at rest than during exercise.

\section{Methods}

\section{Participants}

The 16 participants (7 females, 9 males) were experienced players (at regional or national level) in decision-making sports (handball, basketball, tennis, soccer). Before taking part in the experiment, all 
participants signed written consent forms and were fully informed about the protocol. The mean $( \pm s)$ physical characteristics of the participants were as follows: age, $22.8 \pm 2.5$ years; maximal oxygen uptake $\left(\dot{V} \mathrm{O}_{2 \max }\right), 47.2 \pm 6.93 \mathrm{ml} \cdot \mathrm{kg}^{-1} \cdot \mathrm{min}^{-1}$; maximal heart rate, $186 \pm 9$ beats $\cdot \min ^{-1}$; maximal aerobic power (MAP; the power that elicits $\left.\dot{V} \mathrm{O}_{2 \max }\right), 281 \pm 56.3 \mathrm{~W}$; $20 \% \mathrm{MAP}, 56.3 \pm 11.2 \mathrm{~W} ; 50 \% \mathrm{MAP}, 140 \pm 27.5 \mathrm{~W}$; ventilatory threshold power (the power that elicits the ventilatory threshold), $148 \pm 30.4 \mathrm{~W}$.

\section{Materials}

The participants sat on a cycle ergometer (Ergoline 800S) in front of a computer screen. The choice reaction time task involved operating two levers by hand as quickly and as accurately as possible to a visual stimulus shown in a 200-ms time-frame. The resistance of the levers was controlled by an electromagnetic brake of constant voltage.

Four response signals were randomly presented in the centre of the screen. Each signal corresponded to a specific response, namely a flexing or a stretching of the right or left wrist. Three experimental factors were manipulated: signal quality, stimulus-response compatibility and time uncertainty. Each factor manipulation was crossed with the others.

Response signals were true and false arrows constructed from dots located in a rectangular dotted frame. Stimulus degradation was obtained by removing dots from the frame of the intact stimulus (an arrow) to randomized positions within the frame that were not occupied by the dots of the arrow (see Fig. 2).

Each stimulus could be degraded in four different ways and their order of appearance was randomized to minimize perceptive learning. In the compatible stimulus-response condition, the true arrow indicated the direction and the hand to be used. In the incompatible stimulus-response condition, there was a cross-over relation (side and direction) between the true arrow and the response to be produced. The participant had to respond with the hand and in the direction symmetrically opposed to those indicated by the arrow. In the low time uncertainty condition, the foreperiod duration was a 500-ms fixed interval; in the high time uncertainty condition, the foreperiod duration was a 5000-ms fixed interval.

The inter-trial duration depended on the participant's swiftness to return to the starting position. A new trial began only after a $200-\mathrm{ms}$ stabilization period in the starting position. Knowledge of results was provided at the end of each trial. These results concerned response speed or type of error: anticipation (reaction time $<150 \mathrm{~ms}$ ), extremely slow response (reaction time $>2000 \mathrm{~ms}$ ), decision error (side and/or direction error).

\section{Design and procedure}

The independent variables in this study were intensity of exercise and complexity of the reaction time task. Manipulating the computational factors (signal quality, stimulus-response compatibility, time uncertainty) allowed the complexity of the task to be altered. To distinguish the negative dual-task effect from the facilitating effect of exercise, the participants were tested at rest and at two intensities of exercise: while cycling at $20 \%$ of their maximal aerobic power and while cycling at $50 \%$ of their maximal aerobic power.

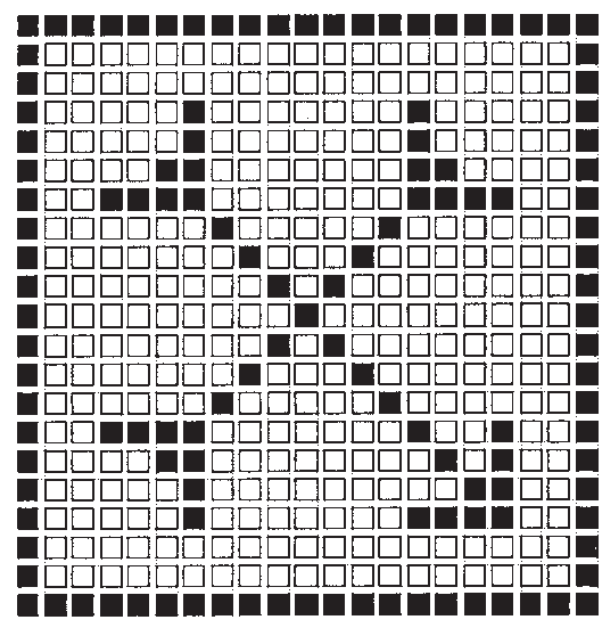

(a)

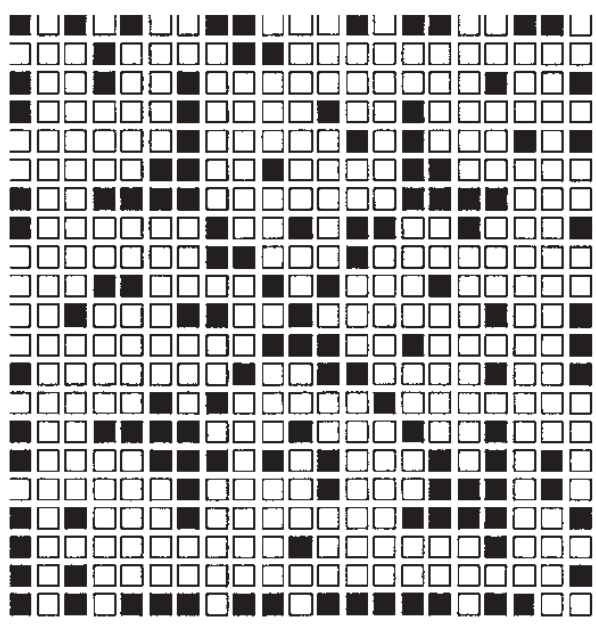

(b)

Fig. 2. (a) Example of an intact stimulus. (b) Example of a degraded stimulus. 
Before taking part in the experiment, maximal oxygen uptake, maximal aerobic power, maximal heart rate and the ventilatory threshold were individually determined using an incremental test to exhaustion. Each participant was required to pedal on the cycle ergometer (Ergoline 800S) until volitional exhaustion. After a warm-up ( $5 \mathrm{~min}$ at $75 \mathrm{~W}$ ) and a rest period ( $3 \mathrm{~min}$ ), the test started at an initial workload of $25 \mathrm{~W}$, with increments of $25 \mathrm{~W}$ each minute until exhaustion (Wasserman et al., 1973). Expired gases were collected and heart rate monitored continuously (CPX, Medical Graphics). The maximal power reached at $\dot{V} \mathrm{O}_{2 \max }$ was recorded.

After this test, the participants were allowed a period of familiarization. They completed the self-evaluation questionnaire, and practised the critical flicker fusion test (Campden Instruments, $12021^{\star} \mathrm{C}$ ) and the choice reaction time task. This learning session minimized the possibility that practice effects would interfere with the effects of physical exercise and increased the output quality of stages during the choice reaction time task.

Then the participants took part, randomly, in three sessions using the same procedure alternation. During each session, the participants performed two choice reaction time blocks of 128 trials (four series of 32 trials) in the morning and in the afternoon.

During the morning, two choice reaction time blocks of 128 trials were presented. The total duration of one block of 128 trials was approximately $17 \mathrm{~min}$. From one block to the other, stimulus-response compatibility was alternated. A rest period was allowed between the two blocks. The first and the second half of each block established two levels of time uncertainty. Within each block, signal quality was varied between eight successive 32-trial parts in alternating order. The order of presentation of the eight 32-trial parts was randomized across the participants. For each participant, the same counterbalanced order was maintained for the three sessions. These morning performances, performed at rest (without pedalling on the cycle ergometer), were recorded as reference data for the day.

The same procedure was used in the afternoon but the participants were, as a function of the days, randomly tested at rest or at one of the two intensities of exercise: while cycling at $20 \%$ and while cycling at $50 \%$ of their maximal aerobic power. The test started with a 3-min warm-up (without performing the choice reaction time task), with frequency of pedalling freely chosen by the participant. Pedalling rate and heart rate were continuously recorded during the simultaneous task (Sport tester systems, Polar, Finland). No knowledge of results about these two variables was provided to the participants. After heart rate and pedalling rate had reached a steady state (after $3 \mathrm{~min}$ of exercise), the simultaneous task started. The participants continued cycling throughout the choice reaction time block of 128 trials. Total exercise time in both conditions was $20 \mathrm{~min}$ ( $3 \mathrm{~min}$ warm-up $+17 \mathrm{~min}$ at the target intensity). Between the two blocks, a rest period was allowed until individual heart rate returned to resting values. The critical flicker fusion test was carried out before and after each block. The participants sat in front of a device that presented a light-emitting diode in front of each eye. The flicker frequency changed: either it increased or it decreased. The participants were required to respond by pressing a button when he or she discriminated, after a foveal fixation, flicker from fusion (and vice versa). They performed three ascending and three descending tests alternately. The average of the six values, in hertz, was used as an overall response. Self-evaluation questionnaires were completed before and after each choice reaction time block. Two self-evaluation questionnaires were used: a French version of the $16-100 \mathrm{~mm}$ visual analogue mood scales proposed by Bond and Lader (1974) and the DP-15 scale (Delignières et al., 1994). This DP-15 scale measured the perceived exertion of the participants.

\section{Data analysis}

The physiological variables (heart rate and pedalling rate) were analysed using a univariate analysis of variance (ANOVA). Post-hoc Newman-Keuls analyses were conducted on all significant interactions. Significance was set at $P<0.05$ for all analyses.

The statistical analyses of reaction time, critical flicker fusion, subjective alertness and perceived exertion were carried out by taking into account baseline performances in order to minimize the effect of intra-individual variability resulting from changes in the participants' mood and psycho-physiological states between the different experimental sessions. Reference performances recorded at rest in the morning of each session served as the covariate in an analysis of covariance (ANCOVA). In addition, orthogonal planned comparisons were performed on the critical flicker fusion and subjective alertness values to assess specific effects of exercise. For each experimental session, a planned comparison (C; vector $2,-1,-1)$ was performed to compare values recorded before and after the choice reaction time task (after the first and the second block of choice reaction time trials).

To confirm the additive effect of signal quality, stimulus-response compatibility and time uncertainty on mean reaction time and variance in reaction time, we conducted an ANOVA on the complete reaction time data (morning plus afternoon reaction time data of each session). 


\section{Results}

\section{Heart rate, pedalling rate and perceived exertion}

Heart rate and pedalling rate data were recorded only during exercise $(20 \%$ and $50 \%$ of maximal aerobic power) and were analysed three times after beginning cycling: between the 6th and 9th minutes, between the 11 th and 14th minutes and, finally, between the 16th and 19th minutes. The heart rate data of five participants were removed from the analysis because of data acquisition failure in one or more experimental conditions. A $2 \times 2 \times 3$ (exercise intensity $\times$ block $\times$ period) ANOVA on the heart rate values revealed a main effect of period $\left(F_{2,20}=34.5\right.$, $P<0.05)$ and a significant interaction between exercise intensity and block $\left(F_{1,10}=6.92, P<0.05\right)$. The main effect of period indicated an increase in heart rate during the 20-min exercise blocks. For the interaction, Newman-Keuls tests indicated that heart rate was significantly higher at $50 \%$ of maximal aerobic power (mean $=145$ beats $\cdot \min ^{-1}$ ) than at $20 \%$ of maximal aerobic power (mean $=100$ beats $\cdot \min ^{-1}$ ). There was no difference in heart rate recorded during the two blocks.

The pedalling rates recorded during exercise were analysed using the same procedure. The pedalling rate data of three participants were not included in the analysis because of data acquisition failure in one or more experimental conditions. The results revealed significant interactions between exercise intensity and period $\left(F_{2,24}=20.8, P<0.05\right)$ and between block and period. Newman-Keuls tests showed that pedalling rate was significantly higher at $50 \%$ of maximal aerobic power $\left(\right.$ mean $\left.=71 \mathrm{rev} \cdot \mathrm{min}^{-1}\right)$ than at $20 \%$ of maximal aerobic power $\left(\right.$ mean $\left.=61 \mathrm{rev} \cdot \mathrm{min}^{-1}\right)$. At $50 \%$ of maximal aerobic power, pedalling rate increased significantly from $70 \mathrm{rev} \cdot \mathrm{min}^{-1}$ between the 6 th and 9 th minutes to $71 \mathrm{rev} \cdot \mathrm{min}^{-1}$ between the 11 th and the 14 th minutes. It reached $73 \mathrm{rev} \cdot \mathrm{min}^{-1}$ between the 16th and 19th minutes. At $20 \%$ of maximal aerobic power, pedalling rate only increased between the first two time frames analysed (6-9 min: $60 \mathrm{rev} \cdot \mathrm{min}^{-1}$; $11-14$ minutes: 61 $\left.\mathrm{rev} \cdot \mathrm{min}^{-1}\right)$. Pedalling rate adjustments were only significant during the first block of exercise.

The effects of exercise intensity on perceived exertion were analysed using a $3 \times 2$ (session $\times$ block) ANCOVA. The analysis revealed a significant main effect of session only $\left(F_{2,28}=13.0, P<0.05 ; d=0.48\right)$. The DP-15 scores increased with exercise intensity. Newman-Keuls test showed that the DP-15 scores were significantly higher at $20 \%$ maximal aerobic power $($ mean $=8.59)$ than at rest $($ mean $=6.69)$, and that it was also significantly higher at $50 \%($ mean $=9.88)$ than at $20 \%$ maximal aerobic power.

\section{Effects of computational variables on mean reaction time, variance in reaction time and errors}

The effects of computational variables on mean reaction time, variance in reaction time and errors were analysed using a $3 \times 2 \times 2 \times 2 \times 2$ (session $\times$ time of the day $x$ signal quality $\times$ stimulus-response compatibility $\times$ time uncertainty) ANOVA.

The analysis of mean reaction showed significant main effects of all independent variables. Reaction time increased from intact to degraded stimuli (mean $=537$ vs $615 \mathrm{~ms}$ ), from compatible to incompatible stimulusresponse mapping (mean $=518$ vs $634 \mathrm{~ms}$ ) and from short to long foreperiod (mean $=567$ vs $585 \mathrm{~ms}$ ) $\left(F_{1,14}=160.8, P<0.05 ; F_{1,14}=190.2, P<0.05 ;\right.$ and $F_{1,14}=6.32, P<0.05$, respectively). An additive pattern was seen between signal quality (SQ), stimulusresponse compatibility (SRC) and time uncertainty $(\mathrm{TU})\left(\mathrm{SQ} \times \mathrm{SRC}: F_{1,14}=0, P=0.99\right.$; SQ $\times$ TU: $F_{1,14}=0, P=0.94 ;$ SRC $\times$ TU: $F_{1,14}=0.02, P=0.89$; $\left.\mathrm{SQ} \times \mathrm{SRC} \times \mathrm{TU}: F_{1,14}=0, P=0.96\right)$. For mean reaction time variance, there were significant main effects of signal quality and of stimulus-response compatibility $\left(F_{1,14}=84.5, P<0.05\right.$ and $F_{1,14}=32.4$, $P<0.05$, respectively). No significant effect of time uncertainty was observed $\left(F_{1,14}=2.89, P=0.11\right)$. An additive pattern was observed between signal quality, stimulus-response compatibility and time uncertainty $\left(\mathrm{SQ} \times\right.$ SRC: $F_{1,14}=1.88, P=0.19 ;$ SQ $\times$ TU: $F_{1,14}=0.37, P=0.55 ; \quad$ SRC $\times$ TU: $F_{1,14}=0.17$, $\left.P=0.68 ; \mathrm{SQ} \times \mathrm{SRC} \times \mathrm{TU}: F_{1,14}=0.9, P=0.35\right)$.

Percentage data cannot be normally tested by ANOVA as their means and variances are closely related. However, the arcsine transform is efficient in stabilizing the variances of these data (Winer, 1970). All percentages were therefore transformed accordingly before being submitted to ANOVA. The arcsine transformed proportion of decision errors showed significant effects of stimulus-response compatibility $\left(F_{1,14}=8.22, P<0.05\right)$. Errors increased when participants were confronted with an incompatible stimulusresponse relationship. We did not observe effects of signal quality and time uncertainty on the proportion of errors $\left(F_{1,14}=0.7, P=0.42\right.$ and $\left.F_{1,14}=0.16, P=0.70\right)$.

\section{Critical flicker fusion and subjective feelings}

The stimulating effect of exercise on critical flicker fusion values and on the participants' subjective states was analysed using $3 \times 3$ (session $\times$ block) ANCOVA. For each session, planned comparisons were performed to compare critical flicker fusion values recorded before and after the two blocks of the choice reaction time task. 
The analysis of critical flicker fusion values showed a significant main effect of session $\left(F_{2,28}=4.02, P<0.05\right)$ and an interaction between session and block $\left(F_{4,56}=4.29, P<0.05\right)$. The critical flicker fusion values were greater at $50 \%$ of maximal aerobic power $($ mean $=34.5 \mathrm{~Hz})$ than at rest $($ mean $=33.8 \mathrm{~Hz})$ and also greater at $50 \%$ than at $20 \%$ of maximal aerobic power $($ mean $=34.0 \mathrm{~Hz}$ ). During the rest session, the comparison revealed a decrease in critical flicker fusion values recorded before $($ mean $=34.2 \mathrm{~Hz})$ and after the choice reaction time task (mean $=33.6 \mathrm{~Hz}$ ) $\left(F_{1,14}=5.89, P<0.05\right)$. In contrast, in the $50 \%$ maximal aerobic power condition, there was an increase in critical flicker fusion values recorded before (mean $=34.1 \mathrm{~Hz}$ ) and after the choice reaction time task (mean $=34.6 \mathrm{~Hz}$ ) $\left(F_{1,14}=10.7, P<0.05\right)$. No difference was observed during the $20 \%$ maximal aerobic power condition $\left(F_{1,14}=1.65, P=0.22\right)$ (Fig. 3).

The stimulating effect of exercise at $50 \%$ of maximal aerobic power was also confirmed by the participants' subjective state of alertness. The interaction of exercise and block was significant $\left(F_{4,56}=5.74, P<0.05\right)$. Planned comparison revealed an increase in alertness state after exercise at $50 \%$ of maximal aerobic power. Indeed, the alertness rating recorded after the choice reaction time task was greater than that before $\left(F_{1,14}=5.2\right.$, $P<0.05)$. No change was observed either during rest or exercise at $20 \%$ of maximal aerobic power $\left(F_{1,14}=1.24\right.$, $P=0.28$ and $F_{1,14}=0.13, P=0.73$, respectively) (Fig. 3 ).

\section{Effects and localization of physical exercise}

The effects of exercise on mean reaction time, variance in reaction time and proportion of decision errors were analysed using a $3 \times 2 \times 2 \times 2$ (session $\times$ signal quality $\times$ stimulus-response compatibility $\times$ time uncertainty) ANCOVA.

We did not observe an effect of session on arcsine transformed proportion of decision errors, which suggests there was no speed-accuracy trade-off $\left(F_{1,14}=0.59, P=0.56\right)$.

The facilitating effect of exercise on mean reaction time was significant $\left(F_{2,28}=3.87, P<0.05\right)$. Post-hoc analyses show that reaction time was shorter during exercise at $50 \%$ of maximal aerobic power $($ mean $=544 \mathrm{~ms})$ than at rest $($ mean $=564 \mathrm{~ms})$. No significant effect was observed between rest and exercise at $20 \%$ of maximal aerobic power (mean $=555 \mathrm{~ms}$ ), but that between $20 \%$ and $50 \%$ of maximal aerobic power showed a trend towards significance $(P=0.10)$ (Fig. 4). None of the interactions between the effects of physical exercise and manipulation of the computational factors approached significance (session $\times \mathrm{SQ}: \quad F_{1,14}=0.01, \quad P=0.94$; session $\times$ SRC: $F_{1,14}=2.32, P=0.15$; session $\times$ TU: $\left.F_{1,14}=0.01, P=0.94\right)$. We did not observe any difference in variance of $\mathrm{RT}$ as a function of exercise intensity.

\section{Discussion}

The use of an experimental protocol constructed in accordance with the recommendations of Tomporowski and Ellis (1986) and Brisswalter and Legros (1996) allowed us to enhance cognitive efficiency during exercise. In line with previous studies (e.g. Brisswalter et al., 1994; McMorris and Graydon, 1996, 1997; Arcelin et al., 1997, 1998), our results support the
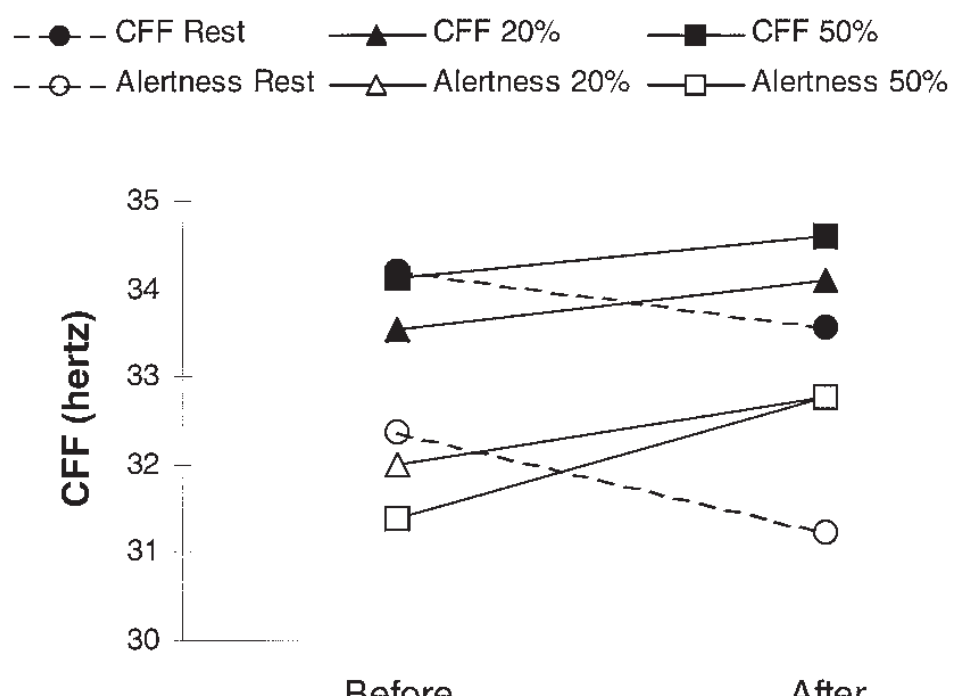

Fig. 3. Change in critical flicker fusion $(\mathrm{CFF})$ and self-perceived alertness state during the experimental sessions as a function of exercise intensity. 


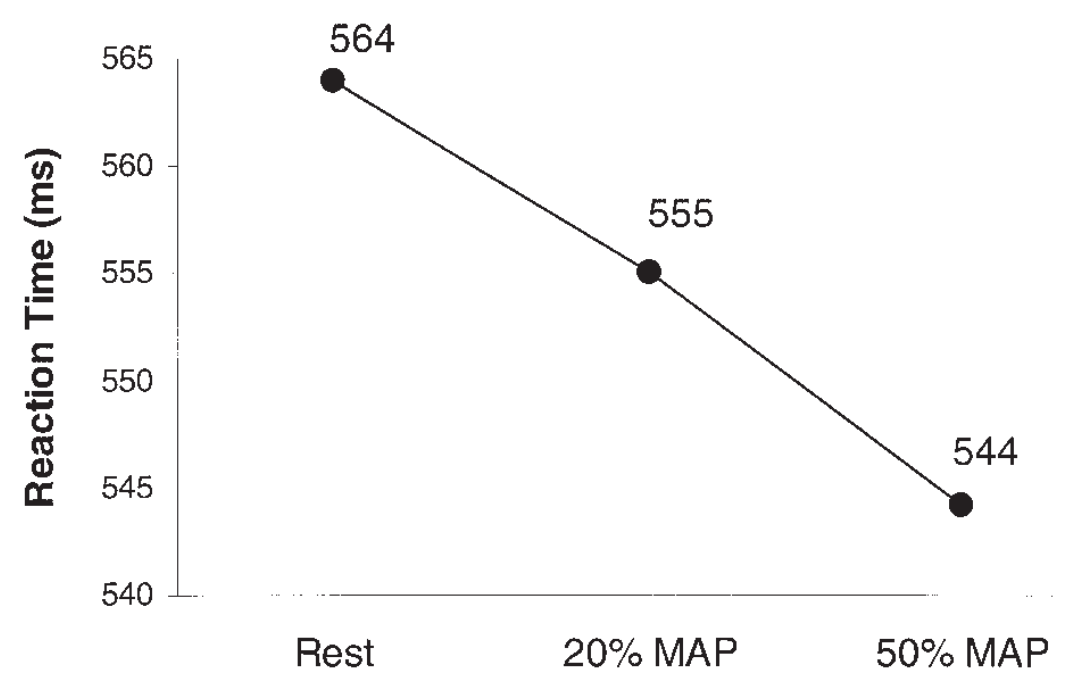

Fig. 4. Change in reaction time as a function of exercise intensity. $\mathrm{MAP}=$ maximal aerobic power.

hypothesis that submaximal exercise $(50 \%$ of maximal aerobic power) improves performance on a choice reaction time task. Indeed, reaction time was $20 \mathrm{~ms}$ faster during exercise at $50 \%$ of maximal aerobic power than at rest.

Despite our prediction, there was no negative effect of a simultaneous task during exercise at $20 \%$ of maximal aerobic power, and no performance impairment was observed. On the contrary, reaction time tended to improve. On the one hand, the absence of a negative dual-task effect on reaction time could be the result of the freely chosen pedalling rate, which was economical and required little concentration (Brisswalter et al., 1995, 2000; Arcelin et al., 1997). On the other, the choice reaction time task and cycling task may not share the same resources. In the resting condition, the critical flicker fusion values were seen to decrease between the tests before and after the choice reaction time task. The boring nature of the task could easily explain this change. The negative effect induced by the repetitive nature of the task, habituation phenomena and central fatigue arising over time could bring brought about the impairment in critical flicker fusion. Unlike at rest, the critical flicker fusion values did not decrease during exercise at $20 \%$ of maximal aerobic power. These results suggest that exercise at $20 \%$ of maximal aerobic power helps to alleviate the boredom associated with the task and allowed the participants to maintain a constant level of arousal.

During exercise at $50 \%$ of maximal aerobic power, there was a positive effect at a behavioural level (mental chronometry) as well as for critical flicker fusion and subjective alertness. All dependent variables recorded in this study highlight the occurrence of physiological and neurophysiological changes that appeared during ex- ercise and induced an improvement in cognitive performances.

The effects of the computational variables in this study were consistent with those observed in the literature on the additive factors method (for a comprehensive review, see Sanders, 1998). The signal quality, stimulus-response compatibility and time uncertainty manipulations caused an increase in reaction time. Moreover, the signal quality and stimulusresponse compatibility manipulations induced an increase in the variance of reaction time. The interaction between signal quality, stimulus-response compatibility and time uncertainty was not significant for mean reaction time or variance in reaction time. According to Sternberg (1969), the additive computational factor effects observed on mean reaction time and on the variance in reaction time suggest that experimental variables affect different processing stages.

Based on the choice reaction time task data, the facilitating effect of exercise was undeniable. Physical exercise improved response speed without any changes in response accuracy. However, no significant interaction was observed between exercise and signal quality, between exercise and stimulus-response compatibility or between exercise and time uncertainty. According to additive factor logic, this lack of interaction suggests that the facilitating effect of exercise does not affect the feature extraction stage, the response selection stage or the motor adjustment stage. Our results were not in line with those of Arcelin et al. (1998), who suggested a facilitating effect of moderate exercise on cognitive functioning mediated by specific influences of activation in the later stages of information processing.

The lack of an interaction between exercise and our computational factor manipulations is at odds with a number of assumptions that predict interactions 
rather than additivities. According to previous studies, the beneficial effect of moderate exercise is probably linked to physiological and neurophysiological changes (for a review, see McMorris and Graydon, 2000). Indeed, an association between the concentration of central catecholamines and central nervous system (CNS) activation in exercising men has been suggested by Peyrin et al. (1987). These authors claimed that mental improvement during exercise may be the consequence of central noradrenergic activation. Moreover, Chmura et al. (1994, 1998) have shown that there is a significant correlation between the concentration of plasma catecholamines and reaction time during exercise in man. Chmura et al. (1994, 1998) claimed that increased concentrations of catecholamines in the central nervous system during exercise should induce an improvement in performance. Based on brain catecholamines, Pagliari and Peyrin (1995a, b) found a positive correlation between central noradrenergic activation during exercise and peripheral adrenaline secretion in rats. As suggested by McGuinness and Pribram (1980), if a close relationship exists between arousal and activation mechanisms and different systems of noradrenergic, cholinergic and dopaminergic neurotransmitters, moderate exercise should probably modulate arousal and/or activation. Therefore, an interaction should exist between exercise and experimental manipulations according to additive factor logic.

The failure to find an interaction in our experiment suggests two alternatives. First, moderate exercise produces additivity at three of the four stages suggested by the (four-stage) model proposed by Sanders (1983), but its effect on the pre-processing stage (influenced by signal intensity) is still unknown. The parallel effect on reaction time and on critical flicker fusion suggests that exercise affects sensory stages and future research should aim to identify the cause of this observation. Second, according to Arcelin et al. (1998), the facilitating effect of exercise should affect the later stage of information processing and the additive factor logic does not allow us to localize this effect. Future research should use electromyography to study the probable facilitating effect of exercise on peripheral motor processes.

Although additive factors logic appears to offer a powerful yet simple method for establishing the relationships between energetic mechanisms and stage processing, several investigations using this framework have failed to uncover any interactions between a given factor and the experimental manipulations (e.g. Fowler et al., 2000; Davranche and Audiffren, 2002). The interpretation of the additive pattern remains difficult to make using this framework (Fowler et al., 2000, 2001; Smulders and van der Molen, 2001).
In conclusion, our results confirm the facilitating effect of moderate exercise on cognitive performance and suggest that exercise at $20 \%$ of maximal aerobic power could help to maintain arousal. The additive effect of physical exercise with the effects of signal quality, stimulus-response compatibility and foreperiod duration suggests that physical exercise spares the stages of feature extraction, response selection and motor adjustment. The exercise effects could modulate non-cognitive processes at a more peripheral level than feature extraction, response selection and motor adjustment. As suggested by the critical flicker fusion results, one of these non-cognitive and peripheral processes could be related to the retino-cortical system. Analyses of response time distributions and electromyography could be complementary methods in identifying stressors that influence energetic mechanisms.

\section{References}

Arcelin, R., Brisswalter, J. and Delignières, D. (1997). Effect of physical exercise duration on decisional performance. Fournal of Human Movement Studies, 32, 123-140.

Arcelin, R., Delignières, D. and Brisswalter, J. (1998). Selective effects of physical exercise on choice reaction processes. Perceptual and Motor Skills, 87, 175-185.

Bond, A. and Lader, M. (1974). The use of analogue scales in rating subjective feeling. British fournal of Medical Psychology, 47, 211-218.

Brisswalter, J. and Legros, P. (1996). Interactions entre les processus physiologiques et cognitifs: modèles théoriques et approche méthodologique. Science E Sports, 11, 71-80.

Brisswalter, J., Legros, P. and Delignières, D. (1994). Interactions entre processus cognitifs et physiologiques. Science et Motricité, 23, 25-31.

Brisswalter, J., Durand, M., Delignières, D. and Legros, P. (1995). Optimal and non-optimal demand in a dual task of pedalling and simple reaction time: effects on energy expenditure and cognitive performance. Fournal of Human Movement Studies, 29, 15-34.

Brisswalter, J., Arcelin, R., Audiffren, M. and Delignières, D. (1997). Influence of physical exercise on simple reaction time: effect of physical fitness. Perceptual and Motor Skills, 85, 1019-1027.

Brisswalter, J., Hausswirth, C., Smith, D., Vercruyssen, F. and Vallier, J.M. (2000). Energetically optimal cadence vs. freely-chosen cadence during cycling: effect of exercise duration. International fournal of Sports Medicine, 21, 6064.

Brisswalter, J., Collardeau, M. and Arcelin, R. (2002). Effects of acute physical exercise characteristics on cognitive performance. Sports Medicine, 32, 555-566.

Chmura, J., Nazar, K. and Kaciuba-Uscilko, H. (1994). Choice reaction time during graded exercise in relation to blood lactate and plasma catecholamine threshold. Fournal of Sports Medicine, 15, 172-176. 
Chmura, J., Krysztofiak, H., Ziemba, A.W., Nazar, K. and Kaciuba-Uscilko, H. (1998). Psychomotor performance during prolonged exercise above and below the blood lactate threshold. European Fournal of Applied Physiology and Occupational Physiology, 77, 77-80.

Davranche, K. and Audiffren, M. (2002). Effects of a low dose of transdermal nicotine on information processing. Nicotine and Tobacco Research, 4, 275-285.

Delignières, D., Brisswalter, J. and Legros, P. (1994). Influence of physical exercise on choice reaction time in sports experts: the mediating role of resource allocation. fournal of Human Movement Studies, 27, 173-188.

Fischer, H.G., Hollmann, W. and De Meirleir, K. (1991). Exercise changes in plasma tryptophan fractions and relationship with prolactin. International fournal of Sports Medicine, 12, 487-489.

Fowler, B., Hofer, K. and Lipitkas, J. (2000). The exhaustive additivity displayed by nitrous oxide has implications for cognitive-energetical theory. Biological Psychology, 52, 161180.

Fowler, B., Hofer, K. and Lipitkas, J. (2001). A new stage and cognitive-energetical theory: a reply to Smulders and van der Molen (2000). Biological Psychology, 50, 233-237.

Guézennec, C.Y. (2000). La fatigue centrale: électrophysiologique ou neurochimique. Science $\mathcal{E}$ Sports, 15, 228-233.

Hagberg, J.M., Mullin, J.P. and Nagle, F.J. (1978). Oxygen consumption during constant-load exercise. Fournal of Applied Physiology, 45, 381-384.

Isaacs, L.D. and Pohlman, R.L. (1991). Effects of exercise intensity on an accompanying timing task. Fournal of Human Movement Studies, 20, 123-131.

McGuinness, D. and Pribram, K. (1980). The neuropsychology of attention: emotional and motivational controls. In The Brain and Psychology (edited by M.C. Wittrock), pp. 95-139. New York: Academic Press.

McMorris, T. and Graydon, J. (1996). The effect of exercise on the decision-making performance of experienced and inexperienced soccer players. Research Quarterly for Exercise and Sport, 67, 109-114.

McMorris, T. and Graydon, J. (1997). The effect of exercise on cognitive performance in soccer-specific tests. Fournal of Sports Sciences, 15, 459-468.

McMorris, T. and Graydon, J. (2000). The effect of incremental exercise on cognitive performance. International fournal of Sport Psychology, 31, 66-81.

McMorris, T. and Keen, P. (1994). Effect of exercise on simple reaction times of recreational athletes. Perceptual and Motor Skills, 78, 123-130.

Pagliari, R. and Peyrin, L. (1995a). Norepinephrine release in the rat frontal cortex under treadmill exercise: a study with microdialysis. Fournal of Applied Physiology, 78, 21212130.

Pagliari, R. and Peyrin, L. (1995b). Physical conditioning in rats influences the central and peripheral catecholamine responses to sustained exercise. European fournal of Applied Physiology and Occupational Physiology, 71, 41-52.
Parkin, C., Fairweather, D.B., Shamsi, Z., Stanley, N. and Hindmarch, I. (1998). The effects of cigarette smoking on overnight performance. Psychopharmacology (Berlin), 136, 172-178.

Peyrin, L., Pequignot, J.M., Lacour, J.R. and Fourcade, J. (1987). Relations between catecholamines or 3-methoxy 4-hydroxy phenylglycol changes and the mental performance under submaximal exercise in men. Psychopharmacology, 93, 188-192.

Powell, R.R. (1982). Technique for differentiating cortical hemispheric activity following exercise. Perceptual and Motor Skills, 54, 923-932.

Pribram, K.H. and McGuinness, D. (1975). Arousal, activation, and effort in the control of attention. Psychology Review, 82, 116-149.

Sanders, A.F. (1980). Stage analysis of reaction process. In Tutorials in Motor Behavior (G.E. Stelmach and J. Requin, eds.), pp. 331-354. Amsterdam: North-Holland.

Sanders, A.F. (1983). Towards a model of stress and human performance. Acta Psychologica, 53, 61-97.

Sanders, A.F. (ed.) (1998). Elements of Human Performance: Reaction Processes and Attention in Human Skill. Mahwah, NJ: Lawrence Erlbaum Associates.

Smith, J.M. and Misiak, H. (1976). Critical flicker frequency (CFF) and psychotropic drugs in normal human subjects: a review. Psychopharmacology, 47, 175-182.

Smulders, F.T. and van der Molen, M.W. (2001). Exhaustive additivity suggests a new stage not an alternative model: a commentary on Fowler, Hofer and Lipitkas (2000). Biological Psychology, 55, 227-237.

Sternberg, S. (1969). The discovery of processing stages: extensions of Donders' method. Acta Psychologica, 30, $276-315$.

Sternberg, S. (1998). Discovering mental processing stages: the method of additive factors. In An Invitation to Cognitive Science, Vol. 4: Methods, Models, and Conceptual Issues (edited by D. Scarborough and S. Sternberg), pp. 703863. Cambridge, MA: MIT Press.

Thayer, R.E. (ed.) (1989). The Biopsychology of Mood and Arousal. New York: Oxford University Press.

Tomporowski, P.D. and Ellis, N.R. (1986). Effects of exercise on cognitive processes: a review. Psychological Bulletin, 99, 338-346.

Wasserman, K., Whipp, B.J., Koyal, S.N. and Beaver, W.L. (1973). Anaerobic threshold and respiratory gas exchange during exercise. Fournal of Applied Physiology, 35, 236-243.

Winer, B.J. (1970). Statistical Principles in Experimental Design. New York: Mc Graw-Hill.

Yagi, Y., Coburn, K.L., Estes, K.M. and Arruda, J.E. (1999). Effects of aerobic exercise and gender on visual and auditory P300, reaction time, and accuracy. European Fournal of Applied Physiology and Occupational Physiology, 80, 402-408. 